\title{
Doing for Learning? Enseignement des arts plastiques et littératie, des apports réciproques
}

\author{
Sylvain Fabre \\ Equipe CIRCEFT-ESCOL, Universités Paris 8 et UPEC, Rue Jean Macé, 94380 Bonneuil sur Marne, \\ France
}

\begin{abstract}
Résumé. l'enseignement des arts plastiques (AP) en France apporte une contribution spécifique au développement de la littératie des élèves. En effet, si les arts du visuel mettent au défi les possibilités du langage, en ce qui concerne l'expression et l'analyse, ils permettent en retour d'en explorer les différentes dimensions. Trois ordres de bénéfice peuvent être dégagés : a) en tant que réalisations de projet, les arts développent l'usage d'outils scripturaires aidant à leur conduite, comme les fiches-projets ou les story-boards. b) Les arts permettent de faire l'expérience de la construction des notions, ainsi que de leur valeur d'outillage de la perception et de l'action (notion de proportion par exemple). c) Enfin nous faisons l'hypothèse que les arts plastiques favorisent le développement de ce que Vygotski nomme le « langage intérieur » par des situations permettant l'expérience des processus visuels et gestuels de l'action Ces trois ordres de bénéfice sont complémentaires : ils approfondissent la compréhension de la littératie en même temps qu'ils aident à clarifier les enjeux didactiques de la discipline.
\end{abstract}

\section{Introduction : la littératie ou les pouvoirs de l'écrit}

Dans quelle mesure l'enseignement artistique peut-il favoriser la littératie des élèves ? Poser cette question nous conduit à interroger la conception de l'art qui est mise en œuvre dans les classe, ainsi que la conception de la littératie qui en résulte. L'enjeu de l'étude est de montrer des apports réciproques, qui nourrissent d'une part le questionnement d'une didactique des arts plastiques, et d'autre part, une meilleure compréhension des enjeux et des modalités de la littératie. Nous considérons que la notion de littératie permet d'élargir le champ des objets didactiques par-delà le « lire-écrire », pour en montrer les effets et en interroger les conditions de développement. Lire et écrire pour " pouvoir réaliser ses objectifs, développer ses connaissances et son potentiel et jouer un rôle actif dans la société » (PISA, 2000) : cette ambition excède la maîtrise instrumentale. Avec Jean-Marie Privat, nous pourrons avancer «qu'il y a littératie quand un groupe humain se meut et agit dans un espace qui organise les corps et les objets du monde selon les ressources fournies par l'écrit et qu'il pratique un usage raisonné et organisé de ces ressources » [1]. L'apport des arts plastiques peut être interrogé à la lumière de cette définition extensive, qui souligne l'articulation entre des activités de manipulation des signes langagiers, des

\footnotetext{
${ }^{a}$ e-mail : sylvain.fabre@u-pec.fr
}

This is an Open Access article distributed under the terms of the Creative Commons Attribution License 4.0, which permits unrestricted use, distribution, and reproduction in any medium, provided the original work is properly cited. 
opérations cognitives et les effets identitaires qui en résultent. Comment rendre compte des processus de développement de la littératie ? Résulte-t-elle seulement de l'alphabétisation, des apprentissages scolaires, ou d'un rapport au monde qui ne se réduit pas à l'écrit ? Jaubert et Rébière [2] montrent la corrélation qui existe entre maîtrise des notions scientifiques, et capacité à produire un discours cohérent : cette corrélation, qui se retrouve d'un écrit sur l'autre chez les élèves, montre l'existence de «postures », dont la formation permet conjointement le développement de l'écrit et de l'acquisition de notions. Dans une perspective semblable, les arts plastiques sont interrogés dans leur spécificité en même temps que leur apport à une formation générale des élèves : dans ce cas, cependant, le langage tient une place paradoxale, puisque les activités qui sont menées relèvent d'une pratique principalement plastique, et non langagière.

\section{2. «Se couper la langue », ou parler pour apprendre ?}

Il faut souligner combien les arts peuvent constituer une résistance envers l'ambition analytique et conceptuelle que représente le langage. La formule d'H. Matisse, « qui veut se donner à la peinture doit commencer à se faire couper la langue » (extrait d'un entretien radiophonique, début 1942) traduit bien le conflit qui peut exister entre une activité artistique attentive à la singularité de l'instant, créative, critique, exploratoire, et un langage perçu comme porteur de la généralité du concept, voire des stéréotypes du langage commun. S'il faut « oublier toutes les roses pour peindre une rose » ( [3], p. 321), comme le dit encore Matisse, s'il faut se confronter à la rose offerte dans l'instant en oubliant toutes les roses que l'on aura vues auparavant, si le motif doit s'imposer dans sa singularité, alors le langage sera tenu en suspicion en tant que vecteur de généralité, occultant la rose présente derrière toutes celles que l'on aura vues précédemment. Les arts contestent la conceptualité inhérente au langage et le mettent au défi de la singularité, de l'instant, de l'émotion.

Mais l'enseignement des arts n'est pas l'art. La méfiance envers le langage qui peut être celle de certains artistes doit être transmise et expliquée. Comme le montre fortement S. Johsua [4], « on n'enseigne pas une pratique, mais un savoir sur la pratique » : l'art ne s'enseigne peut-être pas mais seulement une forme scolarisée des arts, qui est l'objet de la discipline française des « arts plastiques ». En particulier, le langage est un outil indispensable pour transmettre des propositions de pratique qui doivent être comprises par les élèves du point de vue des résultats qu'ils font anticiper, des contraintes qu'elles imposent, et des libertés qu'elles sollicitent. La mise en action des élèves suppose leur capacité à comprendre les consignes et à se les approprier pour en faire des appuis de leur pratique personnelle. Les facteurs qui déterminent la place du langage dans l'enseignement des arts plastiques relèvent donc de facteurs épistémologiques - liés aux spécificités du domaine d'enseignement - mais aussi didactiques, voire institutionnels. Ces derniers résultent de ce que les choix curriculaires résultent pour une part d'un souci de légitimation de la discipline au sein de l'enseignement obligatoire, - souci qui conduit à mettre en avant les apports de la discipline aux autres disciplines et, de manière plus générale, au développement des élèves (« compétences transversales »).

La référence au langagier a donc un statut paradoxal dans l'enseignement des arts plastiques, entre référence massive, dans les documents officiels, à la verbalisation et aux apports langagiers, et pratique réduite dans les activités de classe. Il s'agit de savoir comment un domaine qui paraît mettre le langage en échec, en promouvant des activités de production, peut paradoxalement promouvoir la compréhension et la maîtrise de l'écrit par les élèves. Notre question, « comment les arts plastiques peuvent-ils contribuer à la littératie ? » appelle donc une question complémentaire, celle de savoir comment l'élucidation du langagier aide à une clarification de la didactique disciplinaire.

Pour explorer ces questions croisées, notre étude s'appuie sur une recherche consacrée à l'enseignement des arts plastiques au collège en France, et qui vise à caractériser la discipline dans son projet et ses modalités de mise en œuvre [5]. Elle a été menée pendant deux ans dans quatre classes de collège (en $6^{\circ}$ et $5^{\circ}$ ), par une méthode ethnographique d'observation participante (135 heures 
de cours observées), complétée par des entretiens avec des enseignants et des observations d'autres classes (13 enseignants), ainsi que des entretiens avec des élèves (43). Notre étude interroge les activités d'enseignement proposées et les réactions des élèves. Le cadre théorique emprunte à la didactique en même temps qu'à la sociologie [6]. En nous appuyant sur les travaux de l'équipe ESCOL [7], nous cherchons à nous rendre attentif aux différences d'interprétation des situations par les élèves en fonction de leurs parcours scolaires et sociaux.

Le corpus qui résulte de cette étude, - journal de terrain et transcriptions des entretiens -, est interrogé ici du point de vue des activités langagières et scripturaires mises en œuvre. S'il apparaît que le temps consacré en classe entière aux interactions verbales de compréhension des consignes et de verbalisation de la pratique est très réduit (de l'ordre de moins de $5 \%$ de la durée des observations), il est possible d'analyser les activités d'enseignement en examinant leurs apports possibles à la littératie. Même si ces bénéfices restent peu explicités par les enseignants, le langagier est sollicité d'une manière que l'étude peut clarifier. Trois types d'activité sont analysés, en tant que significatifs de l'enseignement des AP, et dans la mesure où leur complémentarité met en lumière les différentes facettes de la littératie.

\section{La production artistique comme projet}

Une première famille des activités qui mettent en œuvre le langage en arts plastiques concerne l'élaboration de documents préparatoires aux activités plastiques. Par exemple, si les élèves doivent réaliser un court métrage, l'enseignant leur demande de concevoir d'abord un story-board où ils prévoient les différents plans de leur film :

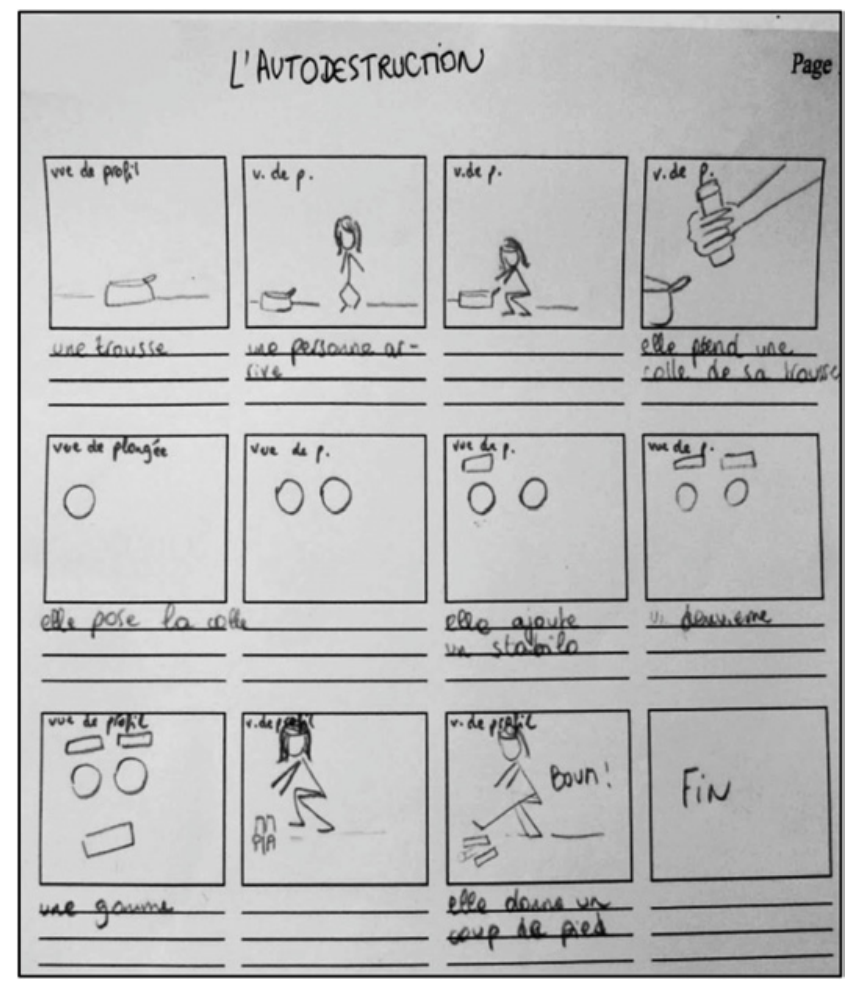

Figure 1. Un exemple de story-board (classe de $5^{\circ}$ - France). 
De même, pour un projet plastique, une fiche-projet est demandée : elle prépare la production en documentant un certain nombre de rubriques. Par exemple, pour un projet donné, l'élève doit indiquer :

1 - la Représentation (figuration par la peinture, la sculpture, le récit, la vidéo etc.)

2 - la Présentation (ou la manière de montrer son travail)

3 - les Techniques (elles dépendent du geste de l'artiste et des outils utilisés)

4 - les Matériaux et leurs qualités (les matériaux ont une histoire et des qualités spécifiques).

Dans chaque cas, la fiche demande d'écrire les opérations prévues, en précisant en quoi les choix répondront à l'énoncé. En quoi ce dispositif favorise-t-il la littératie ? Précisons les apports :

a) La situation peut d'abord être perçue comme un levier motivationnel où les élèves sont incités à écrire pour pouvoir mener une activité plaisante, et parvenir à un résultat gratifiant. La littératie demande une familiarisation longue et répétée à l'écrit, un entraînement continu et intensif où toutes les occasions qui renouvellent l'intérêt des élèves et les incitent à écrire sont profitables. L'apport est donc ici de l'ordre de l'entraînement.

b) Mais, plus fortement, la fiche projet permet de montrer que la production plastique ne se réduit pas à un faire ; elle réduit l'impulsivité et permet l'anticipation. La fiche est un outil scripturaire qui permet la maîtrise de temps par sa spatialisation : le temps du film est maitrisable et manipulable grâce à l'espace de la feuille ; celui de la pratique devient la réalisation d'un projet conçu initialement. Comme l'a montré Goody [8], la « raison graphique » résulte des opérations mentales rendues possibles par l'écrit sous toutes ses formes, et la fiche-projet enrichit les formes graphiques de maîtrise du temps, à l'égal du calendrier ou du planning.

c) Plus précisément, les différentes rubriques constituent un modèle de conception de projet : identification du problème, et élaboration d'une réponse qui consiste en une représentation, par une présentation qui mobilise des techniques et des matériaux. Un savoir-faire de l'organisation de l'action est inculqué grâce à la familiarisation avec ces outils. Les élèves apprennent donc une structure de préparation d'un projet artistique qui contribue à la compréhension de la notion de projet et à sa maîtrise.

d) Enfin, un bénéfice plus spécifique relève du domaine de l'outillage perceptif et de la compréhension de la technique cinématographique : dans le cas du story-board en particulier, le cadre de la fenêtre tracée modélise le cadre de la caméra. La fiche apprend à cadrer, à voir au travers d'une caméra. Le dispositif graphique du cadre prépare à l'usage de cet outil d'enregistrement qu'est la caméra en apprenant à voir au travers d'elle, ou du moins à rendre sensible le fait que la captation d'une scène par une caméra oblige à faire des choix entre des objets dans le champ et hors-champ, à construire la scène en se référant à la géométrie du cadre. De même, les lignes sous le cadre incitent à écrire pour préciser la scène mais aussi pour indiquer des sons, anticiper des jeux de scène, des éléments du décor. Le texte complète l'image en anticipant le film comme ambiance et production fictive de monde (diégèse). Le story-board apprend ainsi le cinéma comme art de l'illusion, c'est-à-dire une technique permettant un registre spécifique d'actions.

On voit donc que les arts plastiques contribuent à la constitution d'un outillage mental qui relève de la littératie. Mais le story-board ou la fiches projets opèrent en minorant la dimension artistique. En effet, les fiches-projets sous-tendent une conception de la pratique comme réalisation d'un projet conçu antérieurement. Le projet s'organise par la définition initiale d'un but, selon un comportement intentionnel qui doit avoir un « haut niveau de conscientisation » comme l'écrit Jean-Pierre Boutinet [9] à propos de la «psychologie des conduites à projet ». Cette recherche d'effectivité et de maîtrise caractérise plus un projet de nature technique qu'artistique. La fiche projet enseigne une méthodologie, c'est-à-dire un savoir-faire qui utilise un programme écrit par avance, qui encadre l'indéterminé du projet par des structures prédéterminées. A l'inverse, les compétences artistiques se caractérisent par 
l'attention à l'imprévu, la capacité à modifier une intention initiale pour prendre en compte les accidents qui peuvent subvenir et qui enrichiront le projet. Il y a «pratique » quand le résultat de l'action ne peut être totalement prévu, quand le faire enrichit l'intention et la déplace : la pratique n'est jamais seulement l'exécution d'un projet préconçu. La fiche semble donc figer le temps au lieu d'en accompagner le développement. L'élève dont on a reproduit le story-board, a trouvé une solution à ce caractère figé en enrichissant sa fiche par l'ajout de mentions complémentaires en rouge (qui portent sur le type de plan souhaité - plan de profil ou en plongée, mentions qui clarifient les dessins et préparent les prises de vue) : mais cette astuce révèle la capacité de cette (bonne) élève à outrepasser le contrat didactique explicite à partir de sa compréhension de la fiche comme outil pour penser - compréhension qui n'est pas donnée dans la fiche même mais a dû être construite antérieurement.

Le dispositif des fiches-projet interroge la spécificité d'un enseignement artistique, entre modèles technologique et artistique, mais aussi la place des outils scripturaires dans une réflexion artistique. L'observation des artistes au travail et l'histoire de l'art montrent de nombreuses traces où l'écrit a une valeur d'aide à la réflexion et à l'élaboration de projets, sous la forme de schémas, croquis, notes, journaux... L'écrit a donc bien une valeur d'aide à l'élaboration de la pensée, ne serait-ce qu'en construisant une instance de distanciation réflexive. Comme le montre Doquet-Lacoste [10] quand elle étudie «la place de l'énonciation dans l'émergence des idées » : «Dans le cas des carnets, des notes, il s'agit d'un échange avec soi-même, soi écrivant à soi qui lira par la suite, avec des contraintes interlocutives (s'adresser à soi plus tard en des termes clairs) et référentielles (nommer précisément). » Les écrits permettent le dialogue avec soi sous la forme d'une adresse à un être imaginaire ou futur (le soi lecteur de soi), - adresse qui incite à « nommer précisément », à clarifier la pensée, en favorisant l'élaboration de la réflexion. La question didactique qui se pose alors est celle du choix des formes d'écrit qui favoriseront cette élaboration psychique et la compréhension de la pratique artistique comme projet, c'est-à-dire projection dans un futur anticipé, en même temps qu'attention à l'accidentel et capacité à infléchir l'intention initiale. Si le carnet de croquis, à feuilles blanches, est opératoire dans le cas des artistes en ce qu'il autorise toutes les inscriptions et toutes les organisations spatiales, il ne paraît pas suffisamment explicite pour des élèves : un savoir-faire didactique serait requis pour trouver un équilibre entre une forme trop contrainte (mais qui peut guider les élèves), et une forme plus à même d'accompagner les mouvements de pensée (mais moins directive et explicite).

Ce point de vue didactique éclaire en retour la littératie même, puisque les AP montrent que l'idée générale d'un outillage mental par des moyens de nature scripturaire doit être précisée en fonction des contextes et des usages. Il n'y a pas une écriture mais plusieurs, en fonction des supports et des usages : donc moins une littératie, en général, que des outils scripturaires plurielles. Nous sommes conduit à des cultures disciplinaires plurielles, où l'écrit a une place importante, mais selon des fonctionnements variables qu'il faut savoir distinguer. Les difficultés d'emploi de la fiche projet montrent le développement de la littératie comme une acculturation à des domaines à la fois intellectuels et cognitifs, alors qu'en retour, la conscience de la dimension littéraciée des outils incite à une scolarisation précise et explicite.

\section{L’expérimentation plastique comme compréhension de la valeur pragmatique des concepts}

Un deuxième type d'activité vise la construction des notions plastiques (espace, couleur, valeur, perspective, proportion, etc.) au moyen d'activités de production. Dans ce cas, l'enseignement des arts plastiques favorise la littératie en permettant l'acquisition de notions qui caractérisent les qualités plastiques des images, que ce soit dans leur perception ou pour l'identification d'effets recherchés. Les savoirs notionnels des arts servent donc d'outils pour la compréhension du monde et la conduite des actions ; c'est dans le cours même de l'action que l'on cherchera à les faire construire. 

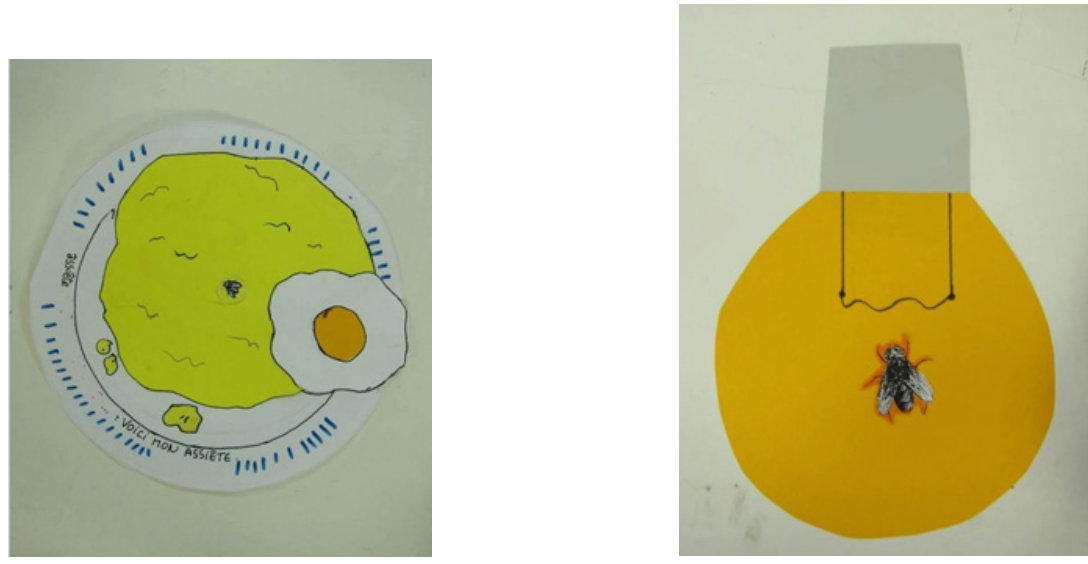

Figure 2. Des mouches bien proportionnées.

Par exemple, l'enseignant veut faire travailler les élèves sur la notion de proportion : lorsque l'on représente (par copie ou invention), il faut que le rapport entre les différents objets représentés soit « bien proportionné » pour produire une illusion de réalisme. Cette illusion exige que les dimensions des différents motifs représentés soient cohérentes entre elles. Comment enseigner cette notion en tant qu'aide pour l'action ? L'enseignant ne la définit pas apriori, mais cherche à la faire apparaître comme un besoin pour une production. Il distribue par exemple la photocopie d'une mouche et demande aux élèves de réaliser une production où ils doivent insérer la mouche et qui doit être réaliste. Après ce premier essai, la photocopie d'une mouche beaucoup plus grande est distribuée, avec la même demande que la production soit réaliste (voir Fig. 2).

Dans la Fig. 2, un élève réalise un collage représentant une omelette sur laquelle la mouche, « petite », est collée, puis une lampe où la mouche agrandie est collée, donnant l'impression qu'on voit la lampe de plus près que l'omelette.En cherchant à produire une image réaliste, les élèves sont conduits, quand ils passent d'une taille de mouche à l'autre, à se rendre compte que tous les objets doivent changer de taille, et qu'il y a une proportion constante entre les différentes dimensions des figures représentées. L'enjeu de cette approche est donc de construire des notions abstraites en tant que repères pour l'action. Ce projet rejoint celui que l'on rencontre en didactique professionnelle [11] pour l'acquisition de concepts qui sont utilisés dans les actions sans être nécessairement explicités. Selon les auteurs, ces concepts se construisent dans l'action et ont une valeur organisatrice de celle-ci, dans la mesure où ils permettent d'identifier dans quelle classe de situations un acteur se trouve. Les notions plastiques sont donc expérimentées d'abord comme des concepts en acte, avant d'être formulées : la notion de proportion aide à repérer un type de problème (quelle taille donner aux différents objets et comment les placer les uns par rapport aux autres), et à agir en fonction des critères qui en résultent. Dans l'organisation préconisée en France pour le cours d'arts plastiques, un temps de verbalisation succède au temps de pratique individuelle et permet de faire réfléchir les élèves sur leurs productions pour rendre conscientes les notions qu'ils ont employées et pour les formuler.

L'apport à une conceptualité organisatrice de l'action est donc original, mais le détour didactique ne va pas sans difficultés. Il n'est pas certain d'abord que les élèves rencontrent implicitement les notions dans leur pratique : le plaisir du faire et du dessiner peut l'emporter sur le questionnement concernant la représentation et les proportions. Parfois, le sujet est seulement l'occasion d'exercer le plaisir de dessiner, et la mouche n'occupe qu'une place marginale, en s'ajustant plus ou moins bien au dessin réalisé indépendamment d'elle. Il faut que la discipline soit conçue comme un lieu d'interrogation 
des activités spontanées, et de conceptualisation de celles-ci, pour que la production soit interprétée comme une expérimentation. Le dessin doit sortir de la sphère du loisir pour devenir objet d'étude et d'interrogation. De même, lorsqu'il y a verbalisation, en classe, le passage du faire au dire ne va pas sans difficulté : il demande que les élèves reconnaissent dans le faire la mise en œuvre implicite d'une notion, qu'ils s'intéressent à celle-ci, en même temps qu'au passage entre le faire et le dire, c'est-à-dire à la manière dont un mot peut produire des effets et dont des actes sont tacitement déterminés par des concepts invisibles que la situation scolaire aide à révéler. Or nos observations nous montrent combien les élèves sont inégaux dans ce qui est à la fois rapport au langage, aux pratiques artistiques et à l'Ecole. Entre des élèves qui utilisent surtout le langage comme moyen de connivence entre pairs et vecteur d'expression émotionnelle, et des élèves pour qui le langage est beaucoup plus un objet de réflexion et de mise à distance du ressenti, le dispositif d'enseignement des arts plastiques accentue les écarts en permettant des malentendus sociocognitifs [12], p. [7-14] quant aux enjeux et aux modalités de travail dans la discipline. Le risque qui apparaît souvent est que le travail notionnel soit vécu comme un apprentissage lexical disjoint de la pratique, et que les élèves aient le sentiment de devoir apprendre des mots « qui ne servent à rien ».

Le travail en arts plastiques peut donc développer la littératie en montrant le pouvoir du langage de décrire le monde et de procurer des outils de caractérisation et de guidage de l'action. Il suppose cependant un rapport au monde suffisamment littéracié pour en comprendre les déterminations et avoir la possibilité de le saisir et de le conceptualiser. Or nos observations des activités des élèves en classe, mis en rapport avec les entretiens, nous donnent à penser que les différences concernent à la fois le sens attribué à la pratique plastique, l'implication pour prendre la parole, et la disposition à considérer le langage comme outil de pensée et d'action. Ce sont le plus souvent les mêmes «bons » élèves qui proposent des productions qui répondent aux consignes et qui verbalisent leurs actions. Ces observations conduisent même à un soupçon réel quant aux finalités de la discipline : les tâches scolaires, en donnant le primat au langagier et au notionnel, ne risquent-elles pas d'empêcher certains élèves de s'investir dans les activités plastiques? Ne constituent-elles pas une forme trop scolarisée d'enseignement artistique qui se coupe de l'important, à savoir le plaisir de produire et la capacité de l'élève de laisser des traces dans lesquelles il se reconnaisse? A vouloir viser des finalités langagières en même temps que de pratique, ne risque-t-on pas d'échouer sur les deux plans, en ne permettant pas aux élèves d'accéder aux enjeux de la discipline ni de développer leur littératie, faute d'une compréhension suffisante de la place du langage dans les activités proposées?

Cette inquiétude conduit à une réflexion didactique : comment organiser les activités pour travailler ensemble la pratique et les notions, quelles trajectoires d'acculturation proposer qui fassent identifier les notions plastiques comme une aide à la réflexion sur l'action? Quelles consignes de pratique plastique à la fois motivantes et porteuses de réflexion proposer ? Une réponse à ces questions peut s'appuyer dans l'idée de communauté de pratique en même temps que langagière, chez Jaubert et Rébière [13]; l'idée de « communauté d'élèves en voie d'instruction » souligne la complémentarité entre pratiques langagières, actions et identités au sein de la classe. La référence à la littératie peut permettre ainsi une relance de la didactique des AP qui concilie ses tensions constitutives et en montre la complémentarité. Néanmoins, cette communauté suppose la constitution de références communes, mais aussi la conscience par chaque élève de pouvoir s'approprier les situations de production et s'y investir. La communauté discursive est aussi mise en commun de pouvoirs d'action individuels, conformément à l'idée d'une littératie, et la dimension langagière doit s'étayer d'une conscience de l'action qui rende nécessaire son outillage pour l'élève. Si l'on considère la subjectivité des acteurs avant leur appartenance à la classe, et leur dimension corporelle complémentaire des usages du langage, un troisième ordre d'expérience peut être mis en évidence, qui porte sur le fonctionnement psychique même. Certaines pratiques des AP visent l'activité mentale dans ses aspects les plus élémentaires, à l'origine des processus perceptifs et moteurs, comme un point de départ pour les constructions notionnelles et l'emploi d'outils scripturaires. 

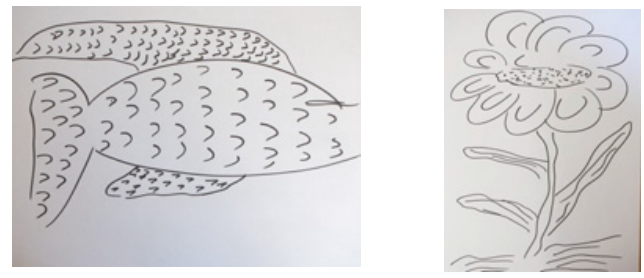

$1 /$ Chaque élève dessine le motif de son choix.

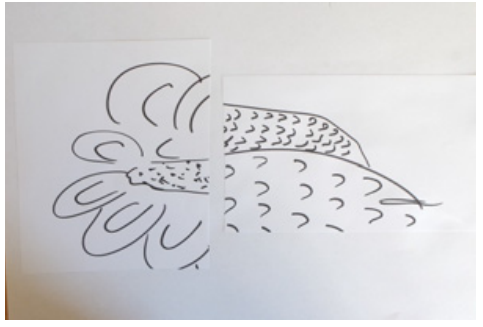

2/ Les morceaux sont découpés et redistribués.

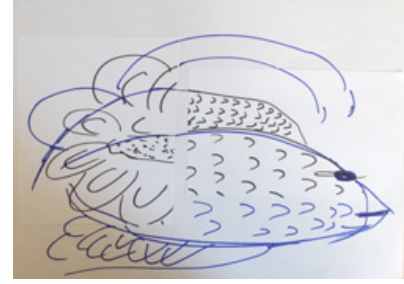

3/ Production d'un nouveau dessin en utilisant les traces données.

Figure 3. Une pratique plastique.

\section{L'agir plastique et l'activité mentale}

Le troisième type d'activité que nous pouvons mettre en évidence reprend les deux précédents apports, concernant le guidage de l'action et sa caractérisation notionnelle. Le niveau d'action change cependant : les activités d'enseignement visent en effet l'agir dans son décours temporel, au plus près du fonctionnement psychique perceptif et moteur. C'est la dimension de pouvoir grâce à une activité scripturaire qui est mise en avant ici. Par exemple, l'enseignant demande d'abord aux élèves de peindre ou dessiner un animal ou un objet, en suscitant leur implication ; dans un deuxième temps, les productions sont découpées et les morceaux redistribués aux élèves. Ceux-ci doivent alors utiliser les morceaux pour produire un nouvel animal : voir Fig. 3.

Cette activité a une forte valeur motivationnelle, puisque les élèves peuvent choisir ce qu'ils vont représenter et qu'ils sont donc incités à dessiner. Mais elle est surtout remarquable par ses effets formateurs : elle apprend en effet aux élèves à regarder une image non seulement en tant que représentation mais aussi dans sa matérialité, en appréciant le rapport entre les traces (les traits dessinés, considérés séparément) et l'effet représentatif que ces traces produisent. Dans la troisième phase en particulier, - à laquelle préparent les deux premières -, le dispositif propose un travail du regard et du geste qui doit opérer une médiation entre la diversité des traces visibles et l'idée du motif que l'on veut représenter : elle enseigne le principe énoncé par Picasso, selon qui « il faut avoir une idée de ce qu'on va faire, mais une idée vague » [[14], p. 121]. Le film de H.-G. Clouzot, Le Mystère Picasso, montre bien, par exemple, comment la peinture de Picasso progresse en s'appuyant sur des tracés qui ne prennent leur dimension représentative que tardivement, en étant d'abord des objets colorés situés dans l'espace. Le tableau avance donc en s'appuyant sur un projet initial vague ainsi que sur l'ensemble des objets plastiques (surfaces colorées, traits) qui effectuent ce projet en même temps qu'ils le modifient. Si l'on compare cette situation avec les fiches-projets étudiés précédemment, on voit combien la maîtrise temporelle s'apprend au cours de la pratique plastique elle-même et non plus comme un préalable à celle-ci. Le dispositif d'enseignement permet à l'élève de prendre conscience du processus de production, par lequel ses gestes répondent à la fois aux traces qu'il perçoit, à l'image plus ou moins vague qu'il se donnait initialement, et à l'image en formation qu'il anticipe au vu de 
sa production en cours. L'élève est appelé à se voir faire en même temps qu'il fait, et donc à prendre conscience des mécanismes de l'action dans ses dimensions temporelles, perceptives et cognitives. La maîtrise du temps ne s'acquiert plus par la disjonction entre projet et réalisation, mais comme conscience de la durée de l'action dans son développement.

Nous avancerons qu'une dimension de la littératie est à l'œuvre dans le pilotage de l'action, sous l'effet de tracés qui sont les objets de l'action en même temps qu'un guide pour celle-ci. Certes, les signes tracés ne sont pas des graphèmes, mais ils ont un pouvoir de sémiotisation, en orientant la pensée vers la représentation d'un objet possible. Entre trait sans signification et pictogramme en cours de formation, ils permettent d'expérimenter le pouvoir de représentation et d'éveil de la pensée propre à tout écrit. C'est l'aspect le plus corporel de la littératie qui est ici sollicité. Il intervient au cœur des processus perceptifs mêmes. Le dispositif manifeste en effet une maîtrise de l'environnement par la compréhension de l'émergence des formes à partir du continuum visible. La psychologie de l'art d'A. Ehrenzweig [15] montre bien comment le processus de création opère par rupture de la perception habituelle où « au niveau de la conscience, la contrainte de la gestalt nous fait diviser le champ visuel en « figure » signifiante et en «fond» insignifiant» (p. 55). Le travail perceptif provoqué par la situation de production à partir de traces préexistantes oblige à interroger ce qui est signifiant et ce qui ne l'est pas, à rompre la dichotomie propre à la gestalt entre figure et fond. L'activité graphique, l'acte d'ajouter des traits aux traits existants pour produire des formes et en inventer de nouvelles, développe une conscience qui relève de la littératie en tant que maîtrise du processus de sémiotisation. L'art élabore et enrichit les modes de sémiotisation du monde, c'est-à-dire les processus de représentation du monde au moyen de formes appartenant à la culture. Elle n'impose pas tant une sémiotisation propre à la littératie (comme les tableaux des fiches projets ou des story-boards) qu'elle fait expérimenter le phénomène même de la sémiotisation, comme organisation de formes qui représentent le réel visible. Certes, cette conscience de la formation de la gestalt reste pour une grande part implicite et en acte. Elle s'éprouve dans la satisfaction du résultat, entre l'idée initiale et la réélaboration de cette idée au cours du travail. Dans une perspective langagière, on pourra considérer qu'elle relève de l'épilinguistique, et non du métalinguistique : la notion d'épilinguistique, proposée par A. Culioli [16], donne à penser la maîtrise en acte qu'ont les sujets, et à quoi la linguistique se confronte pour tenter d'en expliciter la logique. L'exemple des arts plastiques montre donc une « littératie émergente » en tant que non conceptualisée, et même que mouvement de constitution des formes. L'hypothèse d'une continuité entre épilinguistique et métalinguistique, entre littératie émergente et littératie linguistique paraît intéressante, même si elle appelle des études expérimentales à long terme qui paraissent difficiles à mener.

Un autre ordre d'apport de ces pratiques concerne non plus seulement la prise de conscience du fonctionnement perceptif, mais plus largement, le développement du «langage privé », tel qu'on peut le comprendre après Vygotski [17]. Cette notion, qui recoupe la fois le langage égocentrique et le langage intérieur [18], manifeste la manière dont le langage est un instrument essentiel de la médiation de la pensée. C'est par cette activité à la fois psychique et langagière que le sujet peut réguler et planifier son activité - grâce à ce langage, la régulation devient plus indépendante du champ immédiat de la perception. De manière significative, les études expérimentales de Vygotski s'appuyaient sur le dessin, quand il provoquait une situation où « les enfants augmentaient leur quantité de langage égocentrique lorsque leur activité se voyait en difficulté ou obstruée (par exemple lorsque l'enfant se préparait à dessiner et se trouvait sans s'y attendre sans papier ni crayon) » (cité in [18], p. 129). Cette situation, où un enfant anticipe une action de dessin mais en est empêché, est similaire à celles où il dessine en devant utiliser les tracés qui lui sont proposés : entre l'action comme un tout, anticipée globalement, et ce qui vient interrompre cette totalité fonctionnelle, l'évidence se rompt et le langage intérieur est mobilisé comme aide à une action dont l'évidence est interrompue. Le dessin permettrait donc d'expérimenter et de développer la fonction du langage intérieur, pour « réguler et planifier l'activité » (ibid.). Peut-on considérer la pratique artistique comme un levier pour expérimenter et développer le fonctionnement du langage intérieur? Cette hypothèse éclairerait sous un jour nouveau la formule célèbre de Picasso selon 
laquelle : « À huit ans, je dessinais comme Raphaël, mais il m'a fallu toute une vie pour apprendre à dessiner comme un enfant» [[19], p. 13]. Loin de n'être que l'expression d'une idéologie de l'enfance, cette formule pourrait manifester le travail nécessaire pour dépasser une pensée figée, aussi brillante soit-elle, et expérimenter d'autres modes de pensée, par une régression maîtrisée vers des processus originels d'instauration de la pensée, grâce à un accès à des « instruments internes et subjectifs de la relation d'un sujet avec lui-même » (Riviere, ibid). La maîtrise de l'artiste résulterait de cette capacité à expérimenter, revivifier et modifier ce fonctionnement psychique originel.

Lors de la mise en œuvre dans les classes, les activités de « dessin à partir de fragments » ne sont pas sans rencontrer des résistances : les élèves manifestent souvent une déception, voire une certaine colère, quand l'enseignant découpe des productions dont ils sont fiers, et il est difficile de leur montrer que le résultat après reprise sera plus intéressant et plus fort que leur production initiale. Cette résistance est l'objet d'un travail et constitue un enjeu pour la construction de la culture disciplinaire spécifique : cet enjeu est d'importance, puisqu'il porte sur le sens même d'une éducation artistique comme capacité à dépasser les représentations stéréotypées et à rechercher d'autres formes. Une autre difficulté tient au fait que la situation ne suffit pas à déterminer l'activité mentale : les élèves peuvent être submergés par les fragments qu'on leur propose et ne pas y trouver des appuis pour l'activité imageante attendue ; de même, ils peuvent commencer l'activité, en ne comprenant pas que la forme se constitue au cours de l'activité graphique et que c'est en commençant à faire des traits que des amorces de formes se proposeront. Cette difficulté n'est pas étonnante : dans son ouvrage, Ehrenzweig (p. 161-166) souligne bien la résistance des élèves à l'activité de dissociation des formes et au regard de balayage qui permet de produire des formes nouvelles. L'enjeu didactique serait ici d'accompagner le processus mental des élèves, de l'étayer par des actions langagières qui aideraient les élèves à s'approprier la situation, à en repérer les paramètres déterminants (diversité des traits et formes émergentes), voire à modéliser l'activité mentale attendue, par exemple par des protocoles de «speak-aloud ». En retour, il semble que le développement de la littératie demande de s'appuyer sur une pluralité d'activités graphiques, ainsi que sur la complémentarité entre oral et écrit, l'accompagnement proche étant une condition à la formation d'un outillage de pilotage autonome des activités.

\section{Conclusion : des apports réciproques pour une clarification du projet éducatif de l'Ecole}

Comment une discipline qui favorise la pratique, et qui se réfère à un domaine où le langage est suspecté de trahir sa spécificité, peut-elle développer une maîtrise des activités langagières ? Notre étude des pratiques d'enseignement nous a permis de restituer au langagier son importance dans la discipline en montrant ses différentes facettes. Elle met en évidence trois ordres d'apport au développement de la littératie :

a) Le développement d'un outillage scripturaire spécifique. Les arts plastiques utilisent des genres d'écrit que les élèves apprennent à maîtriser, comme ceux permettant la conduite des projets. La littératie se construit donc ici par l'extension du répertoire des types d'écrit utilisés.

b) Le développement d'un contrôle de l'action par un appareil notionnel qui assure la compréhension des propriétés visuelles de l'environnement, et aide à agir sur celui-ci. L'action devient progressivement plus maîtrisée, par une littératie qui se précise comme usage du langage pour agir et acquérir du pouvoir.

c) Enfin un développement de la conscience en acte des processus perceptifs de formation des gestalts par des activités de production, de rupture, et de remaniement, ainsi que le développement du langage intérieur nourri par l'activité de tracé ainsi que l'observation et la sémiotisation des traces. Entre le voir et le faire, entre le dire et le faire, entre continuum visuel et sémiotisation, la pratique plastique est incitation à la pensée sous l'effet de l'écrit comme ensemble de l'activité de tracé et des traces produites. 
Ces trois axes sont complémentaires et montrent l'extension de la notion de littératie, qui est en même temps maîtrise d'un outillage scripturaire, exercice de formes de pensée de description et d'analyse de l'action et du monde s'appuyant sur un travail notionnel, et acquisition de pouvoir par la possibilité de contrôler sa pensée et son action. Nous avons donc souligné l'intérêt d'un dialogue entre la discipline et des préoccupations langagières, que cette notion de littératie permet de penser. L'apport des arts plastiques pourrait être alors de montrer que la littératie, en tant que pouvoir, s'inscrit à même les corps, au plus près de leur action dans l'espace et au moyen d'outils. Ils manifestent l'activité psychique depuis ses composantes les plus «fondamentales » (dynamisme perceptif et moteur) jusque ses usages les plus «élaborés » (pilotage de projets par des outils techniques complexes); et cette pluralité peut apparaître comme un levier pour une progression.

En retour, l'éclairage qu'apporte le point de vue langagier permet une clarification des enjeux des AP ; il conduit à une classification des activités en fonction des activités langagières sollicitées et de la manière de les faire travailler. Une approche didactique peut ainsi émerger par une compréhension clarifiée de la place du langage dans la discipline : entre activités de communication, analyse de la pratique et pilotage de l'action, et développement du langage intérieur. La reconnaissance de ce dernier point pourrait d'ailleurs concilier l'intuition fondamentale d'une résistance des arts au langage avec la reconnaissance du langagier. Dans cet éclairage réciproque, c'est bien le projet éducatif du collège qui peut se préciser, entre apports disciplinaires et recherche de compétences transversales, -respect des différents champs de pratique ou de savoir, et compréhension des voies de développement des élèves.

\section{Références}

[1] J.-M. Privat, Un bain de littératie. À l'école de la piscine. ethnographiques.org, 20 (2010)

[2] M. Jaubert, M. Rebière, Observer l'activité langagière des élèves en sciences. Aster $\mathrm{N}^{\circ} 31$ « 173195 (2000)

[3] D. Fourcade, Henri Matisse. Écrits et propos sur l'art (Ed. Hermann, Paris, 1992)

[4] S. Johsua, Le concept de transposition didactique n'est-il propre qu'aux mathématiques ? In Raisky C. et Caillot M., Au-delà des didactiques, le didactique (de Boeck, Bruxelles, 1996)

[5] S. Fabre, Enseignement des arts plastiques au collège : approche disciplinaire et perspectives normatives en éducation artistique et culturelle (Thèse de Doctorat, Université Paris 8, 2013)

[6] B. Lahire, S. Johsua. Pour une didactique sociologique. Éducation et Sociétés 4/2, 29-43 (1999)

[7] Jean-Yves Rochex, Le Sens de l'expérience scolaire (PUF, Paris, 1995)

[8] J. Goody, La Raison graphique : la domestication de la pensée sauvage, (Éditions de Minuit, Paris, 1978)

[9] J.-P. Boutinet, Psychologie des conduites à projet (PUF, Paris, 2011)

[10] C. Doquet-Lacoste, Écrits intermédiaires, écritures intermittentes : Carnets, notes, bribes de science. Langage et société, 1/127, 7-22, 2009

[11] P. Pastre, P. Mayen, G. Vergnaud, La didactique professionnelle. Revue française de pédagogie, 154, 145-198 (2006)

[12] É. Bautier, P. Rayou, Les inégalités d'apprentissage (PUF, Paris, 2009)

[13] M. Jaubert, M. Rebière, S'essayer à l'écriture littéraire à l'école élémentaire : un enjeu pour la littéracie. In Barré-De Miniac C., La Littéracie, 113-127 (L’Harmattan, Paris, 2004)

[14] F. de Mèredieu, Le dessin d'enfant (Ed. Blusson, Paris, 1990)

[15] A. Ehrenzweig, L'Ordre caché de l'art : Essai sur la psychologie de l'imagination artistique (Gallimard, coll. TEL, Paris 1975-1982)

[16] A. Culioli, A propos du genre en anglais contemporain Les langues modernes, 62, 38-46 (1968)

[17] Vygotski L., Pensée et langage (la Dispute, Paris, 1985)

[18] A. Riviere, La psychologie de Vygotsky (P. Mardaga, Liège, 1990)

[19] P. Désalmant, Picasso par Picasso, Pensées et anecdotes (Ed. Ramsay, Paris, 1996) 